\title{
EVALUACIÓN DE DOS HÍBRIDOS DE TOMATE CHERRY ( Lycopersicum sculentum Mill ) BAJO AMBIENTE PROTEGIDO EN PURISCAL, COSTA RICA ${ }^{1}$
}

\author{
Carlos Cordero², Jorge Rojas
}

\begin{abstract}
RESUMEN
Se evaluó en ambiente protegido la capacidad de producción de dos híbridos de tomate cherry y las cualidades de los frutos con miras a comercializarlos en San Juan de Puriscal entre octubre del 2004 y abril del 2005. Se utilizó un invernadero de $210 \mathrm{~m}^{2}$ donde se sembraron 180 plantas de cada uno de los híbridos Muscato y Natacha. El invernadero no contó con protección de malla antiáfidos. Se analizaron variables de producción y las características de los frutos mediante pruebas de hipótesis de comparación entre medias. El rendimiento por planta del híbrido Natacha fue de $5,4 \mathrm{~kg}$, y el de Muscato de $3,9 \mathrm{~kg}$ con una diferencia significativa $(p \geq 0,01)$ entre ambas. Las diferencias de producción se pueden deber a que Natacha produjo más racimos por planta y a que sus frutos pesaron en promedio 15,04 g mientras que los del híbrido Muscato alcanzaron solamente 11,95 g, la combinación de los dos factores pudo causar las diferencias en la producción total, ya que, ambos híbridos producen la misma cantidad de frutos por racimo. El mercado en Costa Rica exige frutas con pesos cercanos a $13 \mathrm{~g}$. De las dos variedades, se ajustaron más las frutas de Muscato, por su menor tamaño, a los pesos y diámetros de fruta que se prefieren en el mercado. En el ensayo se utilizó poda dejando sólo un eje por planta, con este sistema las frutas del híbrido Natacha sobrepasan las exigencias del mercado en cuanto a tamaño, sin embargo su potencial de producción es muy alto por lo que se sugiere tratar de reducir el tamaño de sus frutas, para lo que es necesario realizar nuevos estudios dejando dos o tres ejes por planta.
\end{abstract}

Palabras clave: Tomate cherry (Lycopersicum sculentum Mill), características, comercialización, producción, calidad.

\section{INTRODUCCIÓN}

En la zona alta de Puriscal, se está consolidando la producción de hortalizas y su cultivo obedece a la necesidad de sustituir el cultivo de tabaco. Es común encontrar en zonas como San Juan siembras de tomate y chile dulce a cielo abierto, bajo medio protegido o en la modalidad de tapado en la que se coloca un plástico protector por encima de las plantas, pero en cualquiera de ellas se encuentran dificultades para alcanzar buenos precios lo que disminuye la rentabilidad.

En los diagnósticos del 2003 de las actividades productivas de la Dirección Regional Central Sur (INTA 2003), las Agencias de Servicios Agropecuarios de Puriscal y Mora indican que uno de los problemas de la región es la carencia de opciones para diversificar no sólo las

${ }^{1}$ Financiado por FITTACORI.

${ }^{2}$ Instituto Nacional de Innovación y Transferencia en Tecnología Agropecuaria (INTA), Costa Rica.

${ }^{3}$ Ministerio de Agricultura y Ganadería (MAG). Dirección Regional Central Sur, Puriscal, Costa Rica. 
actividades agrícolas tradicionales, sino también la reciente modalidad de producir bajo cubierta plástica.

Uno de los problemas más serios que enfrentan los productores al iniciar procesos de diversificación de las actividades, es la elección del cultivo, la variedad y la época de siembra; la elección del cultivo implica un conocimiento previo del comportamiento agronómico, pero en algunos cultivos la oferta tecnológica es todavía reducida. (O'keeffeSwank 2002). Fuera del cultivo tradicional de tomate y chile a cielo abierto y bajo ambiente protegido es poca la disponibilidad de información con que se cuenta para iniciar proyectos productivos en este tipo de cultivos (Centro de investigación en alimentación y desarrollo 2002).

En la región de Puriscal, se cuenta con informaciones recientes en cuanto a las posibilidades de producción de melón y ayote en invernadero durante el invierno (Cordero y Rojas 2004). Se pretende ampliar la oferta tecnológica con la producción de tomate de los tipos Cherry bajo ambiente protegido, ya que según el análisis previo del mercado, posee un nicho importante por lo que puede ser una alternativa de producción no sólo en invierno sino en el verano. Se escogieron tomates Cherry por las posibilidades de comercialización y también porque es un cultivo que no va a competir con las actividades a cielo abierto que cuentan ya con sus propios problemas.

En el cultivo bajo ambiente protegido en algunas variedades se dan problemas patológicos y de adaptación a condiciones específicas de luz, sobre todo en la época lluviosa en que las tardes son nubladas con lo que disminuye la disponibilidad de luz (Centro de investigación en alimentación y desarrollo 2002); por esta razón, poner a disposición de los productores información sobre el comportamiento de tomates cherry motivó la realización de una investigación cuyo objetivo fue determinar las posibilidades de producción y comercialización de dos variedades de tomate tipo Cherry, bajo ambiente protegido.

\section{MATERIALES Y MÉTODOS}

El ensayo se estableció en San Juan de Puriscal cuya zona de vida se clasifica como Bosque muy húmedo premontano (MAG 1984). El experimento se realizó entre los meses de octubre del 2004 y abril del 2005.

Se utilizó un invernadero de $210 \mathrm{~m}^{2}$, construido con estructuras prefabricadas de hierro galvanizado, con alturas laterales de $2,25 \mathrm{~m}$ y máxima de $4,5 \mathrm{~m}$. En el invernadero se utilizó plástico de $7 \mathrm{~mm}$ de grosor tratado con protección ultravioleta y en la parte más alta se construyó una abertura cenital para reducir la temperatura. La estructura no contó con protección de malla antiáfidos.

Los tratamientos consistieron en dos híbridos de tomate cherry de crecimiento indeterminado de la casa Hazera, identificados como Natacha y Muscato. Las especificaciones de cada híbrido señalaron que sus frutos diferían en cuanto a la forma, Natacha es un híbrido con frutos de mayor tamaño y de forma redondeada, Muscato por su parte tiene forma de uva, sus frutos son pequeños pero de color rojo más intenso que los de Natacha.

De cada híbrido se sembraron en suelo tres hileras con 60 plantas/hilera, la separación entre hileras fue de $1,60 \mathrm{~m}$ y entre plantas $0,30 \mathrm{~m}$. Se utilizó como parcela útil las 60 plantas de la calle central de cada híbrido, en esa hilera se aleatorizaron todas las plantas y se seleccionó una muestra de 15 plantas en las que se midió semanalmente el rendimiento referido a: a- número de frutas/ racimo, b- número de racimos/ planta, c- peso en gramos /racimo y d-kilogramos/planta.

En la totalidad de las plantas que se sembraron de cada híbrido se evaluó: arendimiento total en kilogramo /invernadero y b-número de plantas cosechadas. De cada híbrido se seleccionaron al azar 30 frutas en las que se determinaron los grados brix.

Las variables se analizaron mediante una prueba de hipótesis de comparación directa entre medias, utilizando la T de Student. 
RESULTADOS Y DISCUSIÓN

En el Cuadro 1 se presentan las características de rendimiento que se obtuvieron para los dos híbridos, Natacha superó significativamente $(p \leq 0,01)$ a Muscato en la producción por planta en kilogramos, el número de racimos por planta y en el peso del racimo en gramos; en cuanto al número de frutos por racimo no se encontraron diferencias entre los híbridos. Las diferencias de producción entre los híbridos fueron de $755 \mathrm{~kg}$ por invernadero, lo que resulta económicamente muy significativo si se considera que el precio de venta fue de $₫ 450$ por kilo. Con estos precios las diferencias en el ingreso neto por invernadero fueron de $₫ 339.750,00$. Para O'keeffe-Swank (2002) es necesario seleccionar los mejores materiales para la producción en ambiente protegido debido a que los híbridos tienen diferentes respuestas al medio. Los resultados indican que el híbrido Natacha podría tener mayor potencial de rendimiento productivo y económico cuando el cultivo se conduce en la época que abarcó el ensayo.

Los dos híbridos producen el mismo número de frutos por racimo, la diferencia en el rendimiento por planta se puede deber a que Natacha produce más racimos por planta (Cuadro 1) y también a que sus frutos son más pesados que los del híbrido Muscato lo que se aprecia en la (Figura 1). Aunque los dos híbridos mantienen pesos muy parecidos en las primeras tres cosechas (menos de dos gramos de diferencia), posteriormente la diferencia puede alcanzar hasta 4 gra- mos/fruta en las últimas dos cosechas; se observa además un descenso en el peso de la fruta conforme se avanza en las cosechas. Este efecto puede ser ocasionado por la tendencia que tiene la planta a concentrar la producción en los racimos centrales, y causar así competencia entre las frutas.

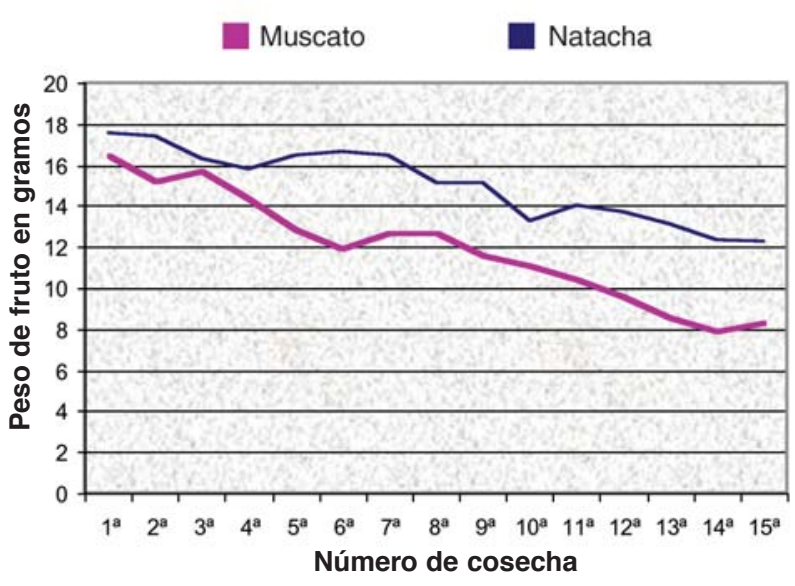

Figura 1. Variación del peso de la fruta según el número de cosecha. Puriscal, Costa Rica. 2004-2005.

En el Cuadro 2 se exponen promedios de algunos aspectos de las características de la fruta en ambos híbridos, se observa que Natacha produjo frutas más grandes tanto en peso como en diámetro que Muscato. El tamaño y peso de la fruta de los tomates cherry en Costa Rica son muy importantes porque los consumidores buscan frutas con tamaños pequeños. Los comerciantes aseguran que los tomates con pesos superiores a 20 gramos son desecho de siembras de tomates corrientes y no del tipo cherry, por eso prefieren frutas con pesos

Cuadro 1. Características del rendimiento de dos híbridos de tomate cherry evaluados en Puriscal entre octubre del 2004 y abril del $2005 .^{1}$

\begin{tabular}{lccccc}
\hline Híbrido & $\begin{array}{c}\text { Producción / } \\
\text { planta en kg }\end{array}$ & $\begin{array}{c}\text { No. de racimos / } \\
\text { planta }\end{array}$ & $\begin{array}{c}\text { Producción } \\
\text { (kg) en } \mathbf{2 1 0} \mathbf{~ m}^{2}\end{array}$ & $\begin{array}{c}\text { No. de frutos / } \\
\text { racimo }\end{array}$ & $\begin{array}{c}\text { Peso del racimo } \\
\text { en g }\end{array}$ \\
\hline NATACHA & $5,418 \pm 0,9155^{\star}$ & $19,0 \pm 0,9790^{\star}$ & $2,709,0$ & 13,6354 & $204,6807 \pm 25,7581^{*}$ \\
MUSCATO & $3,909 \pm 1,3706$ & $17,53 \pm 0,6589$ & $1,954,5$ & 13,0330 & $154,8547 \pm 18,5065$ \\
\hline
\end{tabular}

${ }^{1}$ Los datos son promedios \pm error estándar, con $n=15$.

* Diferencia significativa $(p \geq 0,01)$ para la prueba de hipótesis de comparación entre medias. 
ALCANCES TECNOLÓGICOS, AÑO 4, NÚMERO 1

Cuadro 2. Características de la fruta de dos híbridos de tomate cherry producidos en Puriscal, Costa Rica, 2004-2005.*

\begin{tabular}{lllcc}
\hline Híbrido & $\begin{array}{l}\text { Peso del fruto } \\
\text { en g }\end{array}$ & $\begin{array}{l}\text { Diámetro } \\
\text { de fruto en } \mathbf{~ m m}\end{array}$ & \% de brix & $\begin{array}{l}\text { \% de fruto** } \\
\text { agrietado }\end{array}$ \\
\hline NATACHA & $15,0420 \pm 0,9155(75)$ & $2,7820 \pm 0,044(75)$ & 6,88 & 42,3 \\
MUSCATO & $11,9532 \pm 1,3706(75)$ & $2,3150 \pm 0,059(75)$ & 5,88 & 16,4 \\
\hline
\end{tabular}

* Los datos son promedios \pm error estándar, con $n$ en paréntesis.

** El agrietado de fruto se evaluó después de la $13^{\mathrm{a}}$ corta.

cercanos a los 13 gramos y diámetros con alrededor de 2,85 cm (Incer 2004) ${ }^{1}$.

En las Figuras 2 y 3 se aprecia que la forma de las frutas es diferente. Sobre este aspecto no existen preferencias por los compradores, en lo que si se pone énfasis es en el sabor de las frutas, tienen mejor aceptación las frutas con sabor dulce y bajo nivel de acidez. De los dos híbridos, Natacha tiende a concentrar más grados Brix que Muscato (Cuadro 2) lo cual representa una ventaja a la hora de vender los tomates, sin embargo Natacha es más sensible al agrietamiento de los frutos lo que podría causar pérdidas económicas, si no se varía el manejo agronómico del cultivo en siembras futuras.

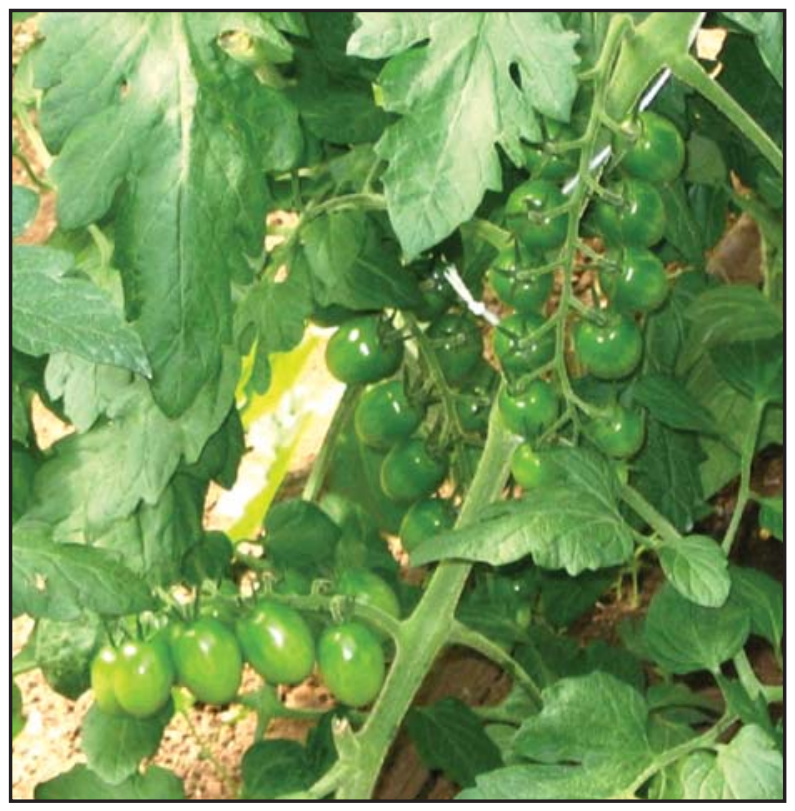

Figura 2. Frutos verdes del híbrido Muscato.

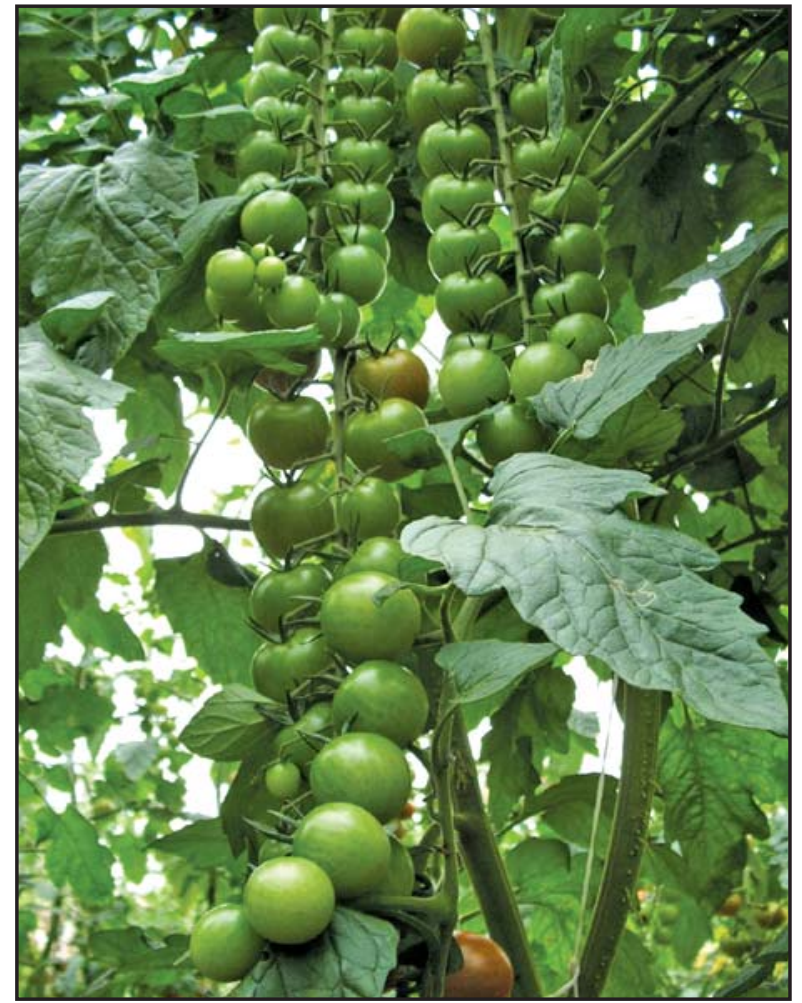

Figura 3. Frutos verdes del híbrido Natacha.

En el ensayo las plantas se podaron bajo el tipo de poda sugerido por Bar-Am (1999) con sólo un eje por planta. Con este sistema, en el híbrido Muscato se cosecharon frutas con pesos promedio muy cercanos a las necesidades del mercado, pero con Natacha sólo se llenaron los requisitos de tamaño en las últimas tres cosechas (Figura 1). Es necesario por tanto, tratar de disminuir el tamaño de la fruta en el híbrido Natacha para lo que se propone aumentar el número de guías o ejes por planta, ya que según Bar-Am

${ }^{1}$ Incer, A . 2004. Tamaños de fruta deseados de tomates cherry. Mercado del CENADA, Barreal de Heredia, Comunicación personal. 
(1999) también podría contribuir a reducir el porcentaje de agrietamiento de los frutos, que se presenta con frecuencia en algunos de los híbridos de tomate cherry.

\section{CONCLUSIONES}

El híbrido Natacha es más productivo que Muscato, se puede obtener hasta $1,5 \mathrm{~kg}$ más por planta, lo que representa $750 \mathrm{~kg}$ más por invernadro (210 m2).

Bajo un sistema de poda de sólo un eje por planta, los frutos del híbrido Natacha tienden a crecer mucho, las dimensiones de la fruta sólo son aptas para el mercado después de la décima semana de cosecha, en tanto que los frutos del híbrido Muscato cumplen con las características del mercado desde la cuarta semana de cosecha.

\section{RECOMENDACIONES}

A pesar que el híbrido Natacha produjo frutas que exceden los tamaños que se prefieren en el mercado local y que además tienden a agrietarse, su alto potencial de rendimiento sugiere intentar producirlo utilizando dos o tres ejes por planta, conservando las mismas densidades de población, con lo que se espera reducir el calibre de los frutos.

\section{LITERATURA CITADA}

Bar-Am Yaacov. 1999. El cultivo en suelo de las variedades de tomates Cherry, bajo condiciones de invernadero. Zeraim gedera Seed Growers Company. Gedera, Israel. sp.

CENTRO DE INVESTIGACIÓN EN ALIMENTACIÓN Y DESARROLLO, A.C. MÉXICO. 2002. Fisiología y Tecnología Poscosecha de Frutas y Hortalizas. Manual de buenas prácticas agrícolas (para frutas y hortalizas frescas). Unidad Culiacán en Fisiología y Tecnología Poscosecha de Frutas y Hortalizas. Talleres Gráficos. México.70p.

Cordero, M.C, Rojas, M.J. 2004 . Manejo agronómico de Cucurbitáceas. In NTA Seminario de hortalizas, Región Central Sur, Región
Pacífico Central ( Puriscal, 6 de octubre 2004). 1 disco compacto.

FAO (Organización de las Naciones Unidas para la Agricultura y la Alimentación, IT).2004. Capítulo 6 Producción Vegetal, 6.1 Cultivos en invernadero Melón(Cucumis melo). (en línea). Consultado el 18 de mayo del 2004. Disponible en http://wwwfao.org /DOCREP/ 005/s86305/s8630s07htm.

INTA (Instituto Nacional de Innovación y Transferencia en Tecnología Agropecuaria, CR). 2003. Diagnóstico de las demandas de tecnología de la Dirección Regional Central Sur (Puriscal) del Ministerio de Agricultura y Ganadería. Puriscal. sp.

MAG ( Ministerio de Agricultura y Ganadería,CR). 1984. Mapa \#5: Ecología y zonas de vida. Adaptado del mapa ecológico de Costa Rica por J.A. Tosi,1969. In Plan de manejo de la cuenca del Río Parrita (Dirección General Forestal), Programa CORENA MAG-AID 515T-032. Esc. 1:200.000.

O'keeffe-Swank, K. 2002. Hay que crear un plan maestro (Puntos clave que incluyen las previsiones para una futura expansión). Productores de Hortalizas (Edición para Centroamérica). (EE.UU.) 4(2):30-34. 
ALCANCES TECNOLÓGICOS, AÑO 4, NÚMERO 1 